\title{
Precision of EBSD based Orientation Measurements
}

\author{
Stuart I. Wright*, Matthew M. Nowell*, and Jay A. Basinger** \\ * EDAX-TSL, 392 E. 12300 S. Draper, UT 84020 \\ ** Dept. of Mechanical Engineering, Brigham Young University, Provo, UT 84604 \\ matt.nowell@ametek.com
}

The precision of orientations measured using automated electron backscatter diffraction (EBSD) has been found to range from approximately $0.5^{\circ}$ to $2^{\circ}[1-4]$. The precision of orientation measurements is important when studying low angle (sub-)grain boundaries as well as plastic deformation. The wide range in angular resolution stated by different authors is due to differences in the methodology used to characterize the angular resolution as well as other factors such as those associated with the Hough transform used to detect the bands, with the camera and collection of the patterns, with indexing and with the pattern center calibration. The two primary factors are the accuracy of the detection of the bands in the EBSD patterns and the accuracy of the pattern center calibration. The impact of some of the band detection parameters has been explored in a previous work [4] with an aim of maintaining angular resolution while maximizing speed. The current work builds upon the earlier work with an aim towards achieving the best precision regardless of speed. Through careful optimization of the parameters involved and new calibration algorithms, we have achieved an order of magnitude improvement in orientation precision. In this work we employ a single crystal to characterize the orientation precision. Using the same set of patterns obtained from a $1 \mathrm{~mm}$ by $1 \mathrm{~mm}$ scan on a silicon single crystal we are able to focus on the effects of band detection and calibration. Some results are shown in Figure 1. In this case the "Standard" is using typical Hough and pattern calibration settings. "Standard+" uses a more accurate method for characterizing the change in the pattern center calibration as the beam moves across the sample during the scan. The curve labeled "Advanced" was obtained using the "Standard+" settings for tracking the pattern center calibration as a function of position in the scan and using an iterative indexing approach based on the work of Kacher et al. [5]. In Kacher et al.'s work the initial estimate is obtained using standard Hough transform based band detection and then simulating the pattern. A comparision is made using crosscorrelation functions [6] between the experimental pattern and the simulated pattern. From the cross-correlation results the pattern is re-simulated and the comparision made again. This iterative approach is taken several times until the simulated pattern matches the experimental pattern as closely as possible. 


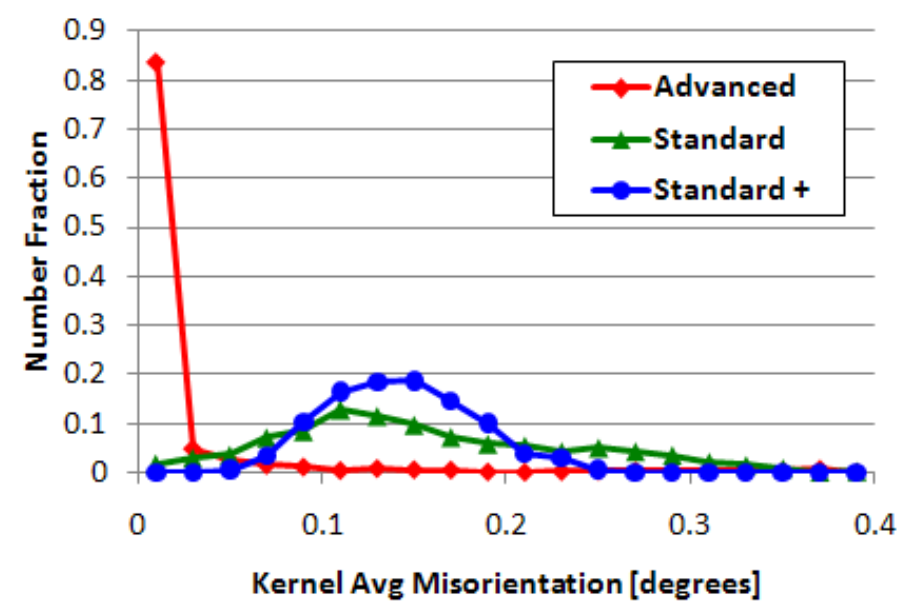

Fig. 1 Orientation spread on single crystal measured using EBSD.

\section{References}

[1] M.C. Demirel, B.S. El-Dasher, B.L. Adams and A.D. Rollett, Electron Backscatter Diffraction in Materials Science, A.J. Schwartz, M. Kumar and B.L. Adams (Eds.), New York: Kluwer Academic/Plenum Publishers, (2000) 65.

[2] F.J. Humphreys, J. Mater. Sci., 36 (2001) 3833.

[3] A. Godfrey, G.L. Wu and Q. Liu, ICOTOM 13, D.N. Lee (Ed.) Seoul: Trans Tech Publications Inc. (2002) 221.

[4] S. Wright and M. Nowell, Adv. Mater. Processes, 166 (2008) 29.

[5] J. Kacher, C. Landon, B.L. Adams and D. Fullwood, Ultramicroscopy, 109 (2009) 148.

[6] A. Wilkinson, Ultramicroscopy 62 (1996) 237. 